\title{
La repetición de la densidad mineral ósea no aportaría información predictiva adicional del riesgo de fractura osteoporótica
}

Repeat bone mineral density screening do not confer additional risk stratification for major osteoporotic fractures

\section{Objetivo}

Determinar si los cambios en la medición de la densidad mineral ósea (DMO) después de cuatro años de una DMO basal proveen información adicional acerca del riesgo de fractura y cuantificar cambios en la clasificación del riesgo después de esta segunda medición.

\section{Diseño, lugar y pacientes}

Estudio de cohortes que incluyó 310 hombres y 492 mujeres que formaron parte del estudio Framingham (Massachusetts, EE.UU.) sobre osteoporosis, con dos mediciones de DMO (cada cuatro años) del cuello femoral tomadas entre 1987 y 1999. Se excluyeron a los participantes que habían tenido una fractura de cadera previa a la realización de la segunda DMO. Se calculó el riesgo fractura osteoporótica (FO) mayor y de cadera durante 2009 o a los 12 años de seguimiento de la segunda DMO. Solo las fracturas de cadera fueron confirmadas con registros en la historia clínica.

\section{Resultados}

La media de edad fue de 74,8 años. La media de variación de la DMO fue $-0,6 \%$ por año (desvío estándar [DE] $1,8 \%$ ). Con una mediana de seguimiento de 9,6 años (rango intercuartil de 5,3 a 12 años) después de la segunda DMO, 76 participantes tuvieron fractura de cadera y 113 FO mayores. Por cada DE de disminución en el porcentaje de cambio anual en la DMO se
Berry SD, y col. JAMA. 2013;310(12):1256-62 observó un aumento en el riesgo de fractura de cadera (HR 1,43; IC95\% 1,16 a 1,78) y FO mayor (HR 1,21; IC95\% 1,01 a $1,45)$ después de ajustar por la DMO basal. A los diez años de seguimiento esto se traduce en un exceso de 3,9 fracturas de cadera por cada 100 personas.

Para evaluar el valor predictivo de la DMO basal y la variación luego de repetir la DMO (a los cuatro años) se diseñaron curvas ROC. La repetición de la DMO no mejoró el desempeño a la hora de predecir fractura de cadera y FO mayor. Una segunda DMO aumentó la proporción de participantes clasificados como de alto riesgo de fractura de cadera en un 3,9\% (IC95\% $-2,2$ a 9,9) y disminuyó la proporción de los clasificados como de bajo riesgo en un $2,2 \%$ (IC $95 \%-4,5$ a 0,1 ).

\section{Conclusiones}

En hombres y mujeres con una media de edad de 75 años que no están en tratamiento para osteoporosis una segunda DMO luego de cuatro años de una DMO basal no mejora significativamente la predicción de fractura de cadera y FO mayor. Repetir la DMO no sería necesario para mejorar la estratificación del riesgo de fractura en esta población.

Fuente de financiamiento: National Institutes of Health; National Heart, Lung, and Blood Institute's Framingham Heart Study; Hebrew SeniorLife.

\section{Comentario}

Actualmente el rastreo de osteoporosis mediante la determinación de la DMO está recomendado en adultos mayores, principalmente en mujeres a partir de los 65 años y solo a edades menores si su riesgo equipara al de una mujer de 65 años ${ }^{1}$. Junto con ciertos factores clínicos de riesgo para fractura (bajo índice de masa corporal, tabaquismo, alcoholismo, consumo crónico de corticoides, etc.) el resultado de la DMO se utiliza en la herramienta FRAX'M (Fracture Risk Assessment Tool) creada por la OMS para evaluar el riesgo a absoluto a diez años de fractura de cadera y fractura osteoporótica (FO) mayor (cadera, columna, antebrazo y hombro) $)^{2,3,4}$.

El valor de repetir la DMO es poco claro. La fuerza de tareas de servicios preventivos estadounidense (en inglés USPSTF) recomienda esperar al menos dos años para repetirla pero remarca que intervalos mayores serían necesarios para mejorar la evaluación del riesgo de fractura' ${ }^{1}$.

Los autores reconocen las siguientes limitaciones del estudio: la mayoría de los participantes realizaron la DMO en dos equipos diferentes, no contaban con información en relación a medicación para la osteoporosis (solo estrógenos, pero por la época se cree que los bifosfonatos no eran utilizados) y falta el antecedente de fractura de cadera en familiar de primer grado para el cálculo del riesgo.

Más allá de esas limitaciones, se destacan como fortalezas el tamaño de la población analizada y el hecho de contar con información longitudinal, lo cual permitió utilizar diferentes modelos estadísticos.

Según las conclusiones obtenidas la repetición de la DMO a los cuatro años provee poca información adicional para la evaluación del riesgo de fractura.

\section{Conclusiones del comentador}

A pesar de que el intervalo apropiado entre DMO sigue sin definirse, la práctica actual más difundida es repetirla cada dos años, lo que no sería necesario en todos los adultos mayores sin tratamiento para OP. El esfuerzo médico podría utilizarse en promover otros factores protectores de las fracturas (y de las caídas que las ocasionan) tales como: incentivar la actividad física, evitar la polifarmacia en ancianos, evaluar la estabilidad y la marcha, mejorar la visión.

Maria de la Nieves Ganiele [ Servicio de Medicina Familiar y Comunitaria del Hospital Italiano de Buenos Aires. maria.ganiele@ hospitalitaliano.org.ar ]

Ganiele MN. La repetición de la densidad mineral ósea no aportaría información predictiva adicional del riesgo de fractura osteoporótica. Evid Actual Pract Ambul. 2015;18(3):90. Jul-Sep. Comentado de: Berry SD, y col. Repeat bone mineral density screening and prediction of hip and major osteoporotic fracture. JAMA. 2013;310(12):1256-62. PMID: 24065012.

Referencias bibliográficas

1. U.S. Preventive Services Task Force. Screening for osteoporosis: U.S. Preventive Services Task Force Recommendation Statement. Ann Intern Med. 2011;154:356-364.

2. World Health Organization Collaborating Centre for Metabolic Bone Diseases. FRAX-WHO fracture risk assessment tool. Reino Unido. Disponible en URL: http://www.shef.ac.uk/FRAX/index.aspx

3. Claus-Hermberg H. y col. FRAX: un nuevo instrumento para calcular el riesgo absoluto de fracturas a 10 años. Medicina (B. Aires) 2009;69:571-575.

4. Kanis J, y col. FRAX ${ }^{\circledast}$ la evaluación de la probabilidad de fractura: introducción. Rev Metab Óseo y Min. 2010;8(1):15-18. 